\title{
Classical and quantum annealing in the median of three-satisfiability
}

\author{
T. Neuhaus, ${ }^{1,2, *}$ M. Peschina, ${ }^{1,3, \dagger}$ K. Michielsen, ${ }^{1, \ddagger}$ and H. De Raedt ${ }^{4, \S}$ \\ ${ }^{1}$ Institute for Advanced Simulation, Jülich Supercomputing Centre, Forschungszentrum Jülich, D-52425 Jülich, Germany \\ ${ }^{2}$ Fakultät für Physik, Universität Bielefeld, D-33501 Bielefeld, Germany \\ ${ }^{3}$ Institut für Kernphysik, Johannes Gutenberg-Universität Mainz, D-55128 Mainz, Germany \\ ${ }^{4}$ Department of Applied Physics, Zernike Institute of Advanced Materials, University of Groningen, Nijenborgh 4, \\ NL-9747 AG Groningen, The Netherlands
}

(Received 20 July 2010; revised manuscript received 10 November 2010; published 18 January 2011)

\begin{abstract}
We determine the classical and quantum complexities of a specific ensemble of three-satisfiability problems with a unique satisfying assignment for up to $N=100$ and 80 variables, respectively. In the classical limit, we employ generalized ensemble techniques and measure the time that a Markovian Monte Carlo process spends in searching classical ground states. In the quantum limit, we determine the maximum finite correlation length along a quantum adiabatic trajectory determined by the linear sweep of the adiabatic control parameter in the Hamiltonian composed of the problem Hamiltonian and the constant transverse field Hamiltonian. In the median of our ensemble, both complexities diverge exponentially with the number of variables. Hence, standard, conventional adiabatic quantum computation fails to reduce the computational complexity to polynomial. Moreover, the growth-rate constant in the quantum limit is 3.8 times as large as the one in the classical limit, making classical fluctuations more beneficial than quantum fluctuations in ground-state searches.
\end{abstract}

DOI: 10.1103/PhysRevA.83.012309 PACS number(s): 03.67.Ac, 02.70.Ss, 64.70.Tg, 75.10.Nr

\section{INTRODUCTION}

At the borderline of mathematics and physics lie optimization problems, which can be cast into solving a minimization problem on a discrete set of variables: Given a scalar cost function $H_{0}(s)$ that is bounded from below by zero, and given a set of integer variables (Ising spins) $s_{i}= \pm 1$ with $i=1, \ldots, N$, one may ask, which assignment or satisfying assignment solves $H_{0}=0$ ? Many satisfying assignment problems with Boolean variables $b_{i}=\left(1+s_{i}\right) / 2$ that are $N P$-hard have precisely this form. In this work, we study the 3-satisfiability (3-SAT) problem, an $N P$-hard problem at the heart of complexity theory [1], by means of methods used in (quantum) statistical physics.

Under the assumption $P \neq N P$, the computational effort for any classical algorithm to solve $N P$-hard problems is believed to be $O\left(e^{g N}\right)$, where $g$ denotes the growth-rate constant. For a trivial classical or unstructured search, that is, the evaluation of $H_{0}$ over all configurations, $g=\ln 2$. In quantum computation, this search finds its analog in Grover's algorithm [2] with $g=\ln 2 / 2$. A polynomial solution to an $N P$-hard problem is expected to have $g=0$.

Conventional, standard adiabatic quantum computation (AQC) $[3,4]$ assumes a linear interpolation between the $N P$ hard problem Hamiltonian $H_{0}$ and a noncommuting driver Hamiltonian $H_{D}=\sum_{i} \sigma_{i}^{x}$ (the "transverse field"), where $\sigma_{i}^{x}$ is the $x$ component of the Pauli matrix. A statistical analysis of AQCs determines the thermodynamic and quantum singularities of the partition function

$$
Z_{\mathrm{AQC}}(\beta, \lambda)=\operatorname{Tr} e^{-\beta\left\{(1-\lambda) H_{D}+\lambda H_{0}\right\}},
$$

\footnotetext{
*t.neuhaus@fz-juelich.de

${ }^{\dagger}$ ma.peschina@fz-juelich.de

${ }^{\ddagger}$ k.michielsen@fz-juelich.de

§h.a.de.raedt@rug.nl
}

where $\beta=1 / k_{B} T$ denotes the inverse temperature, $k_{B}$ is Boltzmann's constant, and $0 \leqslant \lambda \leqslant 1$ is the quantum adiabatic control parameter. In the vicinity of the point $P_{0}^{*}=(\beta, \lambda)=$ $(\infty, 1)$, the optimization problem is solved, as vanishing thermal and quantum fluctuations lead to the exact ground state. We study the approach to $P_{0}^{*}$ from regions of large thermal as well as large quantum fluctuations on lines of parameters $\beta$ and $\lambda$. In particular, we study measures of complexity in the classical limit at $\lambda=1$ as a function of $\beta$ and in the pure quantum limit at almost zero temperature as a function of $\lambda$.

In the classical limit, a measure of complexity is the Monte Carlo (MC) search time for the ground state in a multicanonical ensemble and Wang-Landau simulations [5,6]. These MC simulations perform a Markovian process with random-walk dynamics in the energy. We count the number of MC steps in between ground-state findings in the mean.

In the pure quantum limit, we determine the maximal spin-spin correlation length $\xi_{\max }$, that is, the inverse of the first energy gap at the presumed quantum phase transition at $\lambda^{*}$, from the exponential decay of a two-point function in imaginary time [see Eq. (6)]. If there exists an avoided level crossing, the spin-spin correlation length is finite for a finite number of spins. In accordance with Landau-Zener theory [7], in AQCs the running time of ground-state searches is limited to a time scale $\mathcal{T}$ of order $O\left(\xi_{\max }^{2}\right)$ from below. Hence, for an $N P$-hard problem, a spin-spin correlation length growing exponentially with $N$ would yield a computational complexity for quantum ground-state searches that is similar to the one expected for a classical search, and therefore would make AQC fail.

It is argued that exponentially small energy gaps can be induced by the presence of first-order phase transitions, hampering the performance of AQC in optimization problems related to the 3-SAT problem [8-11]. Their predictive power for specific optimization problems is, however, limited, as any first-order phase transition may-or just may not-turn into 
second order at a critical point. A particularly nasty situation is encountered if there is either a weak first - or second-order phase transition, a situation that has recently been studied in the exact cover problem [12].

\section{THEORY}

The 3-SAT problem is defined on a set of $i=1, \ldots, N$ classical Ising spins $s_{i}= \pm 1$. Its Hamiltonian can be written as a sum of $M$ three-point functions, called clauses,

$$
H_{0}=\sum_{\alpha=1}^{M} \Upsilon^{3}\left(\epsilon_{\alpha, 1} s_{i[\alpha, 1]}, \epsilon_{\alpha, 2} s_{i[\alpha, 2]}, \epsilon_{\alpha, 3} s_{i[\alpha, 3]}\right) .
$$

The function $\Upsilon^{3}$ results from a transcription of the disjunctive cubic Boolean form of a clause $b_{l} \vee b_{k} \vee b_{m}$ to Ising degrees of freedom,

$$
\begin{aligned}
\Upsilon^{3}\left(s_{k}, s_{l}, s_{m}\right)= & \frac{1}{8}\left\{\left(s_{k} s_{l} s_{m}\right)+\left(s_{k} s_{l}+s_{l} s_{m}+s_{k} s_{m}\right)\right. \\
& \left.+\left(s_{k}+s_{l}+s_{m}\right)-1\right\}
\end{aligned}
$$

with $k, l, m \in\{1, \ldots, N\}$ and $k \neq l \neq m$. If $p=1,2,3$ denotes the position of a spin within clause $\alpha$ and if $\eta$ labels an element from a set of $N_{\eta}$ realizations of spins $s_{i}$, the quantity $\epsilon_{\alpha, p}^{\eta}$ takes values \pm 1 . The symbol $i[\alpha, p]$ denotes a map $[\alpha, p] \rightarrow i$ from indices of the clause to the set of spins.

The 3-SAT problem has been studied on random instances with statistical methods, the hardness being characterized by the clauses-to-variables ratio $r=M / N$. It exhibits at least two phases: a "SAT" phase for $r<4.2$ with degenerate ground states, and an "UNSAT" phase for $r>4.2$, where satisfying assignments are exponentially rare [13]. Note that also computationally demanding instances can be constructed for $r=3$ [14].

We study instances with unique satisfying assignments (USA) and for which $r$ is largely free. For this purpose, we generate random ground states $s_{1}^{\mathrm{gs}}, \ldots, s_{N}^{\mathrm{gs}}$ and solve $\epsilon_{\alpha, 1} s_{i[\alpha, 1]}^{\mathrm{gs}}=$ 1 for a particular map $i[\alpha, 1]=\bmod (\alpha-1, N)+1$ and for $\alpha=1, \ldots, M$. The remaining $\epsilon_{\alpha, p}$ and $i[\alpha, p]$ for $p=2,3$ are generated at random with the help of MC updates. We use various filter techniques to exclude non-USA instances.

\section{RESULTS OF SIMULATION}

In studies of the classical limit for $r=5,8$, we use multicanonical ensemble and Wang-Landau simulations [5,6] and determine a statistical estimate of the density of states $n(E)$ on the entire discrete energy interval $0 \leqslant E \leqslant E_{\max }$. We obtain the canonical partition function $Z_{\mathrm{can}}(\beta)$ via its spectral representation and calculate thermodynamic quantities such as the internal energy $\partial_{\beta} \ln Z_{\text {can }}(\beta)$ and the specific heat $\beta^{-2} \partial_{\beta}^{2} \ln Z_{\text {can }}(\beta)$. Numerical analysis shows a discontinuous phase transition at some value $\beta^{*}$, see Fig. 1. Its first-order nature can best be established by considering the ground-state overlap observable $o_{\mathrm{gs}}=N^{-1} \sum_{i=1}^{N} s_{i} s_{i}^{\mathrm{gs}}$ and its distribution function at the specific-heat peak position $\beta_{\mathrm{CMAX}} \approx \beta^{*}$,

$$
P\left(o_{\mathrm{gs}}\right)=Z^{-1} \sum^{\prime} \delta\left(o_{\mathrm{gs}}-\frac{1}{N} \sum_{i=1}^{N} s_{i} s_{i}^{\mathrm{gs}}\right) e^{-\beta_{\mathrm{CMAX}}},
$$

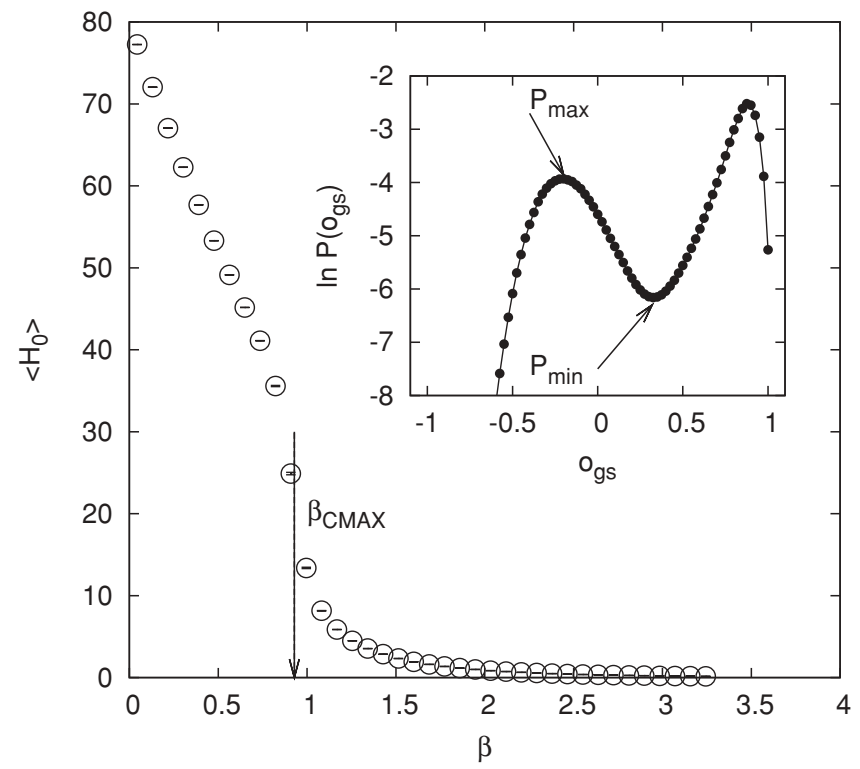

FIG. 1. Classical limit: Expectation value of the problem Hamiltonian $\left\langle H_{0}\right\rangle$ as a function of $\beta$ for a particular realization with $N=80$ and $r=8$. Arrow: Position of the maximal specific heat. Inset: Distribution function of the ground-state overlap observable; see Eq. (4). $P_{\text {max,left }}$ and $P_{\min }$ determine the nucleation barrier $B_{0}$.

where $\sum^{\prime}$ denotes a sum over all spin configurations. For almost all realizations, $P\left(o_{\mathrm{gg}}\right)$ exhibits a bimodal shape with one sharp peak at $o_{\mathrm{gs} \text {,right }} \approx 1$ with a value $P_{\mathrm{max}, \text { right }}$, an example being depicted in the inset of Fig. 1. The second peak with a value $P_{\text {max, left }}$ is well separated from the first one and is located at $o_{\mathrm{gs}, \text { left }}$. At $r=8$, a finite-size scaling analysis yields a nonvanishing overlap order-parameter gap $\left\langle\Delta o_{\mathrm{gs}}\right\rangle=$ $\left\langle o_{\mathrm{gs}, \text { right }}\right\rangle-\left\langle o_{\mathrm{gs}, \mathrm{left}}\right\rangle=1.11$ (1) for the thermodynamic limit and in the mean of realizations. Discontinuous phase transitions are also associated with nucleation free-energy barriers. As the maxima in $P\left(o_{\mathrm{gs}}\right)$ are separated by a minimum with value $P_{\min }$, and in analogy to free-energy barrier definitions in Ising magnets and glasses, we use Binder's method [15] to define a nucleation barrier $B_{0}=\ln \left(P_{\max , \text { left }} / P_{\min }\right):=B_{0}^{\eta}$ for each realization $\eta$.

We also perform $\mathrm{MC}$ simulations in the multicanonical partition function $Z_{\text {muca }}=\sum^{\prime} \exp [-\ln n(E)]$ yielding an equal distribution for the probability $P_{\text {muca }}(E)=$ const to find an energy $E$ in the Markov chain. The MC dynamics in the energy $E$ is different from that of a free random walk with polynomial singularities $\tau \propto N^{2}$ in autocorrelation times, as hidden free-energy barriers at energies in the vicinity of the ground state $E=0$ slow down the diffusion. For each realization we measure the number of local METROPOLIS update steps $\tau_{s}^{0} / N^{2}$ in the mean that an interacting walk in energy spends in the "transition" from $E_{\max }$ toward the ground-state energy. The factor $N^{-2}$ corrects for the trivial free walk behavior that is present even in the absence of barriers.

Figure 2 displays the results for the averages of $\ln \left(\tau_{s}^{0} / N^{2}\right)$ (triangles) and $B_{0}$ (circles) calculated in the median for $r=5,8$ and a number of spins up to $N=100$. Both quantities exhibit exponential behavior of the form $A \exp \left(g_{c} N\right)$ with classical growth-rate constants $g_{c}^{\tau}=0.077$ (4) (barrier) and 


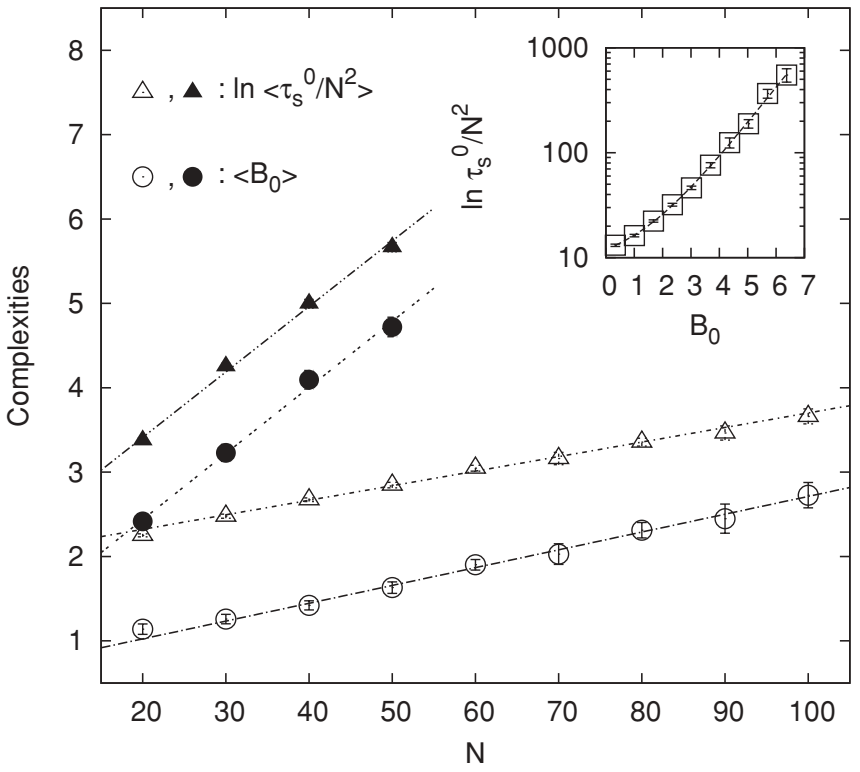

FIG. 2. Classical limit: Expectation values for the logarithmized search time $\ln \left\langle\tau_{s}^{0} / N^{2}\right\rangle$ (triangles) and the nucleation barrier $\left\langle B_{0}\right\rangle$ (circles) as a function of the number of spins $N$. Straight lines correspond to exponential singularities. Open symbols: $r=8$; full symbols: $r=5$. Inset: $N=100$ and $r=8$ dependency of $\ln \left(\tau_{s}^{0} / N^{2}\right)$ on $B_{0}$ within a set of 1000 realizations.

$g_{c}^{B}=0.078(3)$ (search time) for $r=5$. For $r=8$, we find $g_{c}^{\tau}=0.016(1)$ and $g_{c}^{B}=0.021(2)$, respectively. Hence, as expected, the complexity grows as $r$ is lowered to $r^{*} \approx 4.2$ from above. Note, however, that the growth rates are much smaller than $g=\ln 2$ of an unstructured search. The inset of Fig. 2 displays binned mean values of $\tau_{s}^{0} / N^{2}$ as a function of the nucleation barrier $B_{0}$ for $r=8$ and $N=100$ for a set of $N_{\eta}=1000$ realizations. Within this set of realizations, violent fluctuations of complexity-related observables are observed. The search time also shows an exponential behavior of the form $\tau_{s}^{0} / N^{2}=a+b \exp \left(c B_{0}\right)$ with $c \approx 0.8$. Thus the low-temperature free-energy landscape of the 3-SAT problem has the simple property that the static free-energy barrier $B_{0}$ determines the ground-state search dynamics.

We now quantize the problem by introducing a standard Trotter-Suzuki time discretization [16]. We choose a regular temporal lattice with $N_{\tau}=128$ or 256 time slices, a finite step size $\Delta \tau=1$ in the $\tau$ direction, and periodic boundary conditions in Trotter time. The inverse temperature is $\beta=$ $N_{\tau} \Delta \tau$ and the Boltzmann factor of the quantized problem at imaginary time is

$$
\begin{aligned}
\ln \left(P_{B, q}\right)= & -\kappa_{0} \sum_{\tau=1}^{N_{\tau}} H_{0}\left(\left\{s_{1}(\tau), \ldots, s_{N}(\tau)\right\}\right) \\
& -\kappa_{\tau} \sum_{i}^{N} \sum_{\tau=1}^{N_{\tau}} s_{i}(\tau) s_{i}(\tau+1),
\end{aligned}
$$

with positive ferromagnetic hopping parameters $\kappa_{0}=\lambda \Delta \tau$ and $\kappa_{\tau}=-\ln \{\tanh [(1-\lambda) \Delta \tau]\} / 2$ [16]. These equations implement the AQC partition function Eq. (1) as a function of $\lambda$ and $\beta$, up to discretization errors caused by the finiteness of

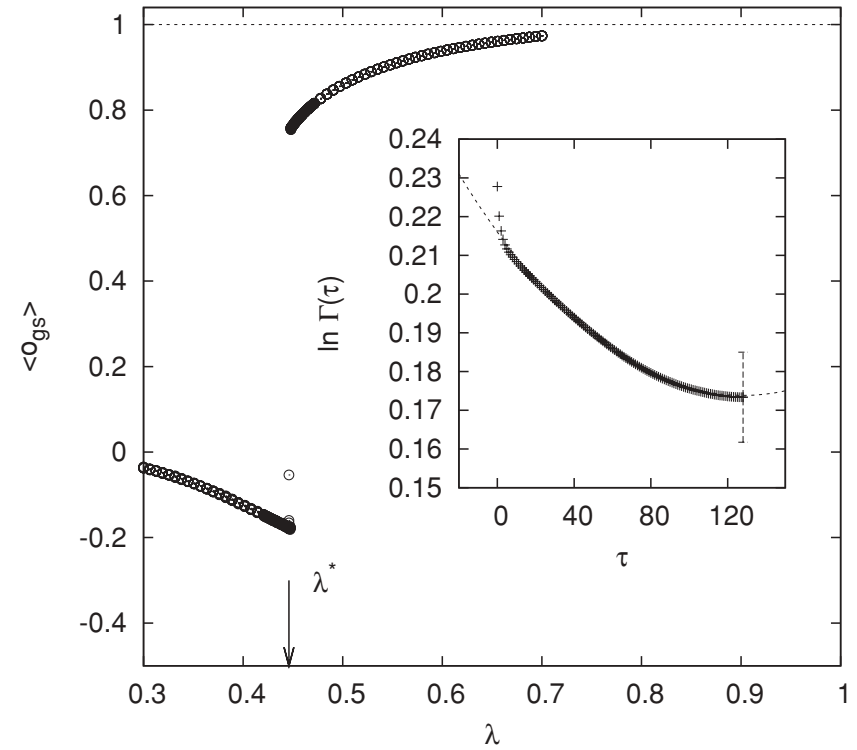

FIG. 3. Quantum limit: Signature of the quantum phase transition for the same realization of Fig. 1. The mean overlap observable $\left\langle o_{\mathrm{gs}}\right\rangle$ as a function of $\lambda$ shows a jump at $\lambda^{*} \approx 0.446$. Inset: Overlap-overlap correlation function $\Gamma(\tau)$ at $\lambda^{*}$ as a function of imaginary time $\tau$.

the regularization. The first energy gap $\xi^{-1}$ is obtained from a large $\tau$ fit to the exponential decay of the expectation value of the connected two-point function in the canonical mean,

$$
\Gamma(\tau)=\langle\mathcal{O}(0) \mathcal{O}(\tau)\rangle-\langle\mathcal{O}(0)\rangle^{2} \propto e^{-\frac{\tau}{\xi}}
$$

We have experimented with several time-local observables $\mathcal{O}(\tau)$ and found that among an extended set of trial observables, the $\tau$-dependent ground-state overlap $o_{\mathrm{gs}}(\tau)=$ $N^{-1} \sum_{i=1}^{N} s_{i}(\tau) s_{i}^{\mathrm{gs}}$ yields the best statistical signals for the exponential decay with $\tau$. The minimum energy gap, or the maximum spin-spin correlation length $\xi_{\max }$, determines the complexity. We find it by a search that uses parallel tempering simulations in the control parameter $\lambda$ [17] on a $\lambda$ partition with 64 elements. Elementary low-temperature exchange updates are absolutely essential for error reduction in the quantum correlator.

We have studied the quantum phase transition of the partition function Eq. (1) for $r=8$ and $N$ values up to $N=80$. In Fig. 3, we display the expectation value of the quantum ground-state overlap observable $\left\langle o_{\mathrm{gs}}\right\rangle=N_{\tau}^{-1} \sum_{N_{\tau}} o_{\mathrm{gs}}(\tau)$ for a specific $N=80$ realization. A quantum phase transition, which is of a blatantly discontinuous nature, is observed at $\lambda^{*}$. Interestingly, the transition to the ground state proceeds with increasing $\lambda$ from configurations that are slightly antiparallel to the known ground state; see the negative values of $o_{\mathrm{gs}}$ for $\lambda<\lambda^{*}$. The inset of Fig. 3 displays the quantum correlation function $\Gamma(\tau)$ of Eq. (6) at $\lambda^{*}$, which for the specific example decays exponentially at the numerical value $\xi_{\max } \approx 185$.

In the analysis of the quantum complexity, we have picked a subset of 91 realizations for $N=60$ at about median-or less-classical complexity given by the $B_{0}$ value. In the inset of Fig. 4, we display the correlation of $B_{0}$ values with their quantum counterparts, the maximal spin-spin correlation length $\xi_{\max }$ values. We observe a linear correlation between 


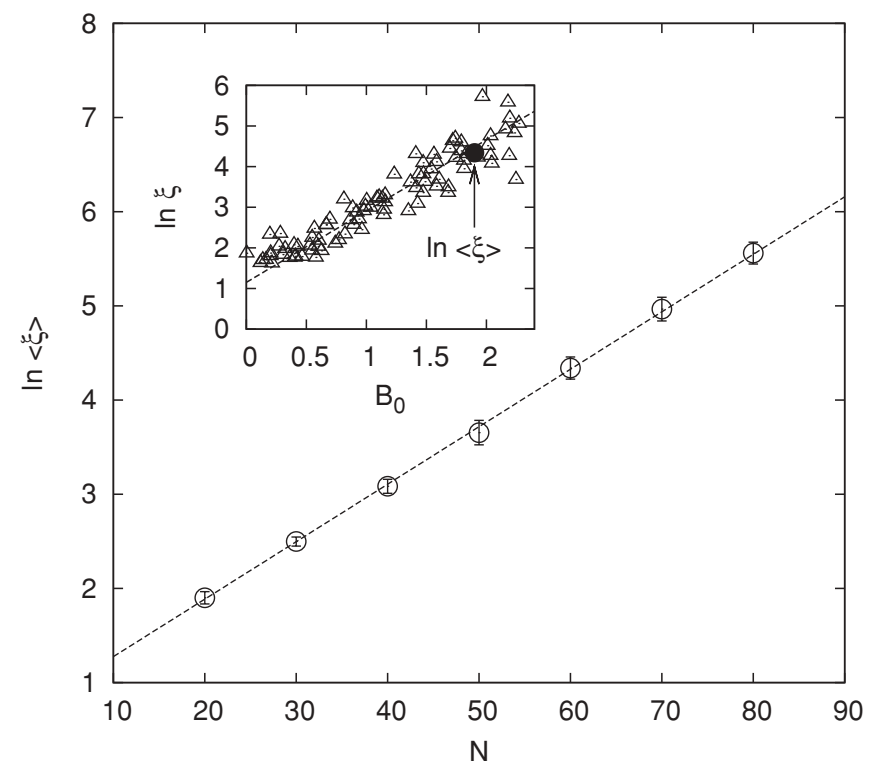

FIG. 4. Quantum limit: Finite-size scaling of $\ln \langle\xi\rangle$ as a function of $N$ for $r=8$. The largest median correlation length is $\langle\xi\rangle=259.9$ at $N=80$. The straight line displays the fit Eq. (7). Inset: Scatter plot of tupels of $\ln \xi$ and $B_{0}$ (triangles) for $N=60$. The data exhibit a linear correlation (straight line). We determine the correlation length at the position of the median barrier $\left\langle B_{0}\right\rangle=1.90(6)$ (arrow and solid circle).

$\ln \xi_{\max }$ and $B_{0}$, see the straight line, and not even the slightest tendency that the quantum complexity is weaker than the corresponding classical one. A similar observation is made for all $N$.

We simplify the calculation of the quantum complexity by use of the median. Note that median averages do not necessarily require the actual calculation of extremal values, but just of those in the vicinity of the median. Given our data, we may determine the spin-spin correlation length $\xi$ at the median position of the nucleation barrier $\left\langle B_{0}\right\rangle$. For correlated data, the latter quantity is a good estimator of the spin-spin correlation length in the median $\langle\xi\rangle$. A fit to the form

$$
\langle\xi\rangle=A e^{g_{q} N},
$$

on the entire $N$ interval $20 \leqslant N \leqslant 80$ with $\chi_{\text {d.o.f. }}=0.1$ (where d.o.f. denotes degrees of freedom), yields for the quantum growth-rate constant $g_{q}=0.061(1)$ for the 3-SAT problem with $r=8$. Figure 4 shows $\ln \langle\xi\rangle$ as a function of $N$ (circles) and the fit in Eq. (7) (straight line). As can be clearly seen, the data are not compatible with polynomial behavior. Following the Landau-Zener theory, twice the growth-rate constant $2 g_{q}$ should be considered for the comparison to the classical search time complexity.

\section{CONCLUSION}

In summary, we have determined the exponential singularities that dominate the classical and quantum running times for ground-state searches in the median of an ensemble in the 3-SAT problem with unique satisfiability assignments. For $r=8$, we measure the growth-rate constants $g_{c}=0.016(1)$ and $g_{q}=0.061(2)$ of the corresponding classical $\tau_{s}^{0}$ and quantum $\xi_{\max }$ exponential behavior in the ground-state searches. Our numerical data are precise and the classical growth-rate constant is confirmed by a static free energy, that is, nucleation barrier $B_{0}$ scaling. The finite-size scaling window is large and excludes a polynomial behavior for quantum and classical searches. For the case of standard AQC, we find that twice the growth-rate constant $2 g_{q}$ undershoots the Grover value $g=\ln 2 / 2$. Hence, standard AQC's constitute a class of ground-state searches that compares favorable to Grover's quantum search. However, there is no indication that for the 3-SAT problem standard AQC's can reduce the exponential complexity to a polynomial one. Thus, standard AQC ground-state searches for $N P$-hard problems are not quite as powerful [18] as was conjectured earlier [4]. We also find a quantum growth-rate constant that exceeds the classical one by a factor of 3.8. Therefore, sole quantum fluctuations are even significantly less efficient than sole classical fluctuations. In the future, one may determine complexities of systems with modified driver Hamiltonians that are optimized with respect to ground-state searches. An alternative quantitative approach to computational intractability can rely on freeenergy calculations for instantons [19].

\section{ACKNOWLEDGMENTS}

T.N. thanks the Theory Department at Bielefeld University for extended hospitality. Calculations were performed on the JUMP and JUROPA supercomputers at JSC and on the NICOLE workstation cluster of NIC (VSR Grant No. JJSC02). This work is partially supported by NCF, the Netherlands.
[1] S. A. Cook, Proceedings of the Third Annual ACM Symposium on the Theory of Computing (ACM, New York, 1971), p. 151.

[2] L. K. Grover, Am. J. Phys. 69, 769 (2001).

[3] T. Kadowaki and H. Nishimori, Phys. Rev. E 58, 5355 (1998).

[4] E. Farhi, J. Goldstone, S. Gutmann, and M. Sipser, e-print arXiv:quant-ph/0001106v1 (2000); E. Farhi, J. Goldstone, S. Gutmann, J. Lapan, A. Lundgren, and D. Preda, Science 292, 472 (2001).

[5] B. A. Berg and T. Neuhaus, Phys. Rev. Lett. 68, 9 (1992).

[6] F. Wang and D. P. Landau, Phys. Rev. Lett. 86, 2050 (2001).
[7] L. D. Landau, Phys. Z. Sowjetunion 2, 46 (1932); C. Zener, Proc. R. Soc. London Ser. A 137, 696 (1932).

[8] R. Schützhold and G. Schaller, Phys. Rev. A 74, 060304(R) (2006).

[9] M. H. S. Amin and V. Choi, Phys. Rev. A 80, 062326 (2009).

[10] T. Jörg, F. Krzakala, G. Semerjian, and F. Zamponi, Phys. Rev. Lett. 104, 207206 (2010).

[11] B. Altshuler, H. Krovi, and J. Roland, Proc. Natl. Acad. Sci. USA 107, 12446 (2010). 
[12] A. P. Young, S. Knysh, and V. N. Smelyanskiy, Phys. Rev. Lett. 101, 170503 (2008); 104, 020502 (2010).

[13] R. Monasson, R. Zecchina, S. Kirkpatrick, B. Selman, and L. Troyansky, Nature (London) 400, 133 (1999).

[14] M. Žnidarič, Phys. Rev. A 71, 062305 (2005).

[15] K. Binder, Phys. Rev. A 25, 1699 (1982).

[16] M. Suzuki, S. Miyashita, and A. Kuroda, Prog. Theor. Phys. 58, 1377 (1977).
[17] D. J. Earl and M. W. Deem, Phys. Chem. Chem. Phys. 7, 3910 (2005), and references therein.

[18] W. van Dam, M. Mosca, and U. Vazirani, How Powerful is Adiabatic Quantum Computation?, in Proceedings of the 42nd Annual Symposium on Foundations of Computer Science (IEEE, New York, 2001), p. 279.

[19] S. Coleman, The Uses of Instantons, in Proceedings of the International School of Subnuclear Physics, Erice, 1977, edited by A. Zichichi (Erice, Italy, 1977). 